\title{
Trampas artesanales con atrayentes alcohólicos una alternativa para el monitoreo y control de la broca del café, Hypothenemus hampei (Ferrari 1867)
}

\section{Crafting traps with attractant alcoholics an alternative for monitoring and control of borer coffee, Hypothenemus hampei (Ferrari 1867)}

\author{
Agramont Richard ${ }^{1 *}$, Cuba Nicanor ${ }^{1}$, Beltrán José Luis ${ }^{1}$, Almanza Juan Carlos $^{1}$, Loza-Murguía Manuel ${ }^{1,2}$
}

\begin{tabular}{|c|c|}
\hline Datos del Articulo & Resumen \\
\hline $\begin{array}{l}{ }^{2} \text { Departamento de Enseñanza e } \\
\text { Investigación en Bioquímica \& } \\
\text { Microbiología-DEI\&BM. Unidad } \\
\text { Académica Campesina Carmen Pampa- } \\
\text { UAC-CP. }\end{array}$ & $\begin{array}{l}\text { con cuatro repeticiones. Se utilizaron tres tipos de trampas artesanales construidas con envases plásticos desechables } \\
\text { de bebidas gaseosas, trampas Casera, Brocap, y Yessica, se evaluaron tres tratamientos: mezcla de alcoholes metílico } \\
\text { (M) y etílico (E) en proporción } 3: 1 \text {; mezcla } 1: 1 \text { de M y E; mezcla 1:1:1 de M, E y cereza fresca de café licuado } \\
\text { (CFCL), y agua como testigo. }\end{array}$ \\
\hline $\begin{array}{l}\text { *Dirección de contacto: Campus } \\
\text { Manning. Unidad Académica } \\
\text { Campesina Carmen Pampa, Coroico, } \\
\text { La Paz Bolivia Casilla 4242 Tel.: } 591 \\
\text { (2) 8781991. E-mail address: } \\
\text { agramont@ } \text { hotmail.com }\end{array}$ & $\begin{array}{l}\text { Las mayores capturas de adultos se presentaron en las trampas caseras con mezclas que contenían mezcla de M-E } 3: 1 \\
\text { con promedios ( } \pm \text { desviación estándar) adultos/trampa/diez días de } 3414.5 \pm 3227.7 \text { ), superando al resto de los } \\
\text { tratamientos. La trampa casera es una de las alternativas para el control y monitoreo de la broca en las plantaciones de } \\
\text { café. }\end{array}$ \\
\hline \multirow[t]{3}{*}{ Palabras clave: } & $\begin{array}{l}\text { alternativa de bajo costo, siendo factible su uso exitoso por parte de los productores en programas de manejo } \\
\text { integrado. }\end{array}$ \\
\hline & (C) 2010. Journal of the Selva Andina Research Society. Bolivia. Todos los derechos reservados. \\
\hline & Abstract \\
\hline $\begin{array}{l}\text { J Selva Andina Res Soc } \\
\text { 2010;1(1):2-12. }\end{array}$ & \multirow{3}{*}{$\begin{array}{l}\text { The necessity to incorporate an alternative, for monitoring and control of the borer coffee, Hypothenemus hampei } \\
\text { (Ferrari) (Choleoptera: Curculionidae) to be feasible for the use of the coffee producers, in the community Choro, } \\
\text { Coripata municipality, second section of Nor Yungas province, La Paz Bolivia. It was evaluated the capture of adult } \\
\text { borer coffee individuals using } 45 \text { traps into } 1.5 \text { hectares distributed at random with four repetitions. It was used three } \\
\text { types of craft traps, built with disposable plastic bottles of soft drinks, with the traps Casera, Brocap and Yessica, } \\
\text { were evaluated three treatments: mixture of alcohols methyl (M) and ethylic (E) in proportions } 3: 1 \text {; mix } 1: 1 \text { of (M) } \\
\text { and (E); mix 1:1:1 of (M) (E) and coffee fresh cherry liquated (CFCL) and water as a witness. } \\
\text { The largest captures of adult individuals, were present in the crafting traps with mixture of (M) (E) } 3: 1 \text { with overalls } \\
\text { ( } \pm \text { standard deviation) adults/traps/ten days of } 3414.5 \pm 3227.7 \text { being superior to the other treatments. The crafting trap } \\
\text { is one of the alternatives for the control and monitoring of the borer in the coffee plantations. } \\
\text { The use of crafting traps with alcoholic attractants for the capture of adult individuals is present as a low cost } \\
\text { alternative, being feasible the successful use by the producers into the management integrated programs }\end{array}$} \\
\hline $\begin{array}{l}\text { Historial del artículo } \\
\text { Recibido Enero } 20,2010 . \\
\text { Devuelto Abril } 18,2010 \\
\text { Aceptado Julio } 30,2010 . \\
\text { Disponible en línea, Octubre } 2010 .\end{array}$ & \\
\hline \multirow[t]{2}{*}{$\begin{array}{l}\text { Crafting traps, } \\
\text { coffee, } \\
\text { Coripata, } \\
\text { pet bottles }\end{array}$} & \\
\hline & (c) 2010. Journal of the Selva Andina Research Society. Bolivian. All rights reserved. \\
\hline
\end{tabular}




\section{Introducción}

La broca del café Hypothenemus hampei (Ferrari) (Coleoptera: Curculionidae: Scolytinae) es considerado el principal insecto plaga en todos los países productores de café; fue descrita por primera vez por el entomólogo austriaco Ferrari en 1867, y en 1903 comenzó a convertirse en una plaga en el cultivo del café, producto del importante repunte que tuvo la siembra de este cultivo en los países del África ecuatorial (LópezVaamonde et al 1997; Damon 2000). Las hembras de la broca perforan el fruto y construyen galerías en su interior, lo cual ocasiona daños como la caída del fruto y pérdida de peso del grano de café (Wegbe et al 2003).

Las plantaciones de café en Bolivia sufren problemas de manejo ineficiente: están bajo densas sombras o sin sombra; en otros lugares los cafetales están envejeciendo. Esta forma de cultivar causa problemas como rendimientos bajos, mayor infestación de plagas y enfermedades (Chuquimia 1996). Una de las plagas que más afecta al café es la broca $H$. hampei, considerada una de las más devastadoras de este cultivo a nivel mundial. Típicamente implica pérdidas importantes en los rendimientos por cosecha que van desde un 5\% hasta un 24\%, según la infestación que se presente. En casos extremos se reportan pérdidas hasta del $50 \%$ de la cosecha (Ramírez \& Mora 2001).

En la región de los Yungas, las mayor incidencia generalmente ocurre en zonas por debajo de los mil metros de altitud con infestaciones que están entre un 20 a 50\% (Cuba 2003). Por otro lado, los daños que se observan no solamente reflejan la rápida propagación de la plaga sino, también, la intensidad de daños directos que ocasiona en los frutos (verdes, maduros). Para combatir la incidencia de la broca, se pueden realizar control de tipo cultural, biológico, etológico y químico. Estas medidas de control son costos adicionales para el productor pero que contrarrestan las perdidas ocasionadas por esta plaga.

En Venezuela, la broca fue reportada por primera vez en 1995 en el estado Táchira y en el 2000 se reportó en el estado Lara en la localidad de Caspo, municipio Andrés Eloy Blanco (Fernández 2005).

En Bolivia la broca del café fue detectada por primera vez en 1978 en Riberalta (Beni), y en abril de 1985 en Caranavi, introducida del Brasil. Desde esta zona se ha diseminado a todas las zonas cafetaleras del país (Belpaire \& Chorquina 1998).

En Colombia, el uso de las trampas de alcoholes se ha venido evaluando bajo un esquema de investigación participativa desde 1998, logrando capturas de hasta 300 hembras de broca por trampa por semana (Aristizábal et al 2002).

En México, Velasco et al (1997) evaluaron el uso de las trampas en períodos inter-cosecha, obteniendo buenos resultados al emplear extractos de la variedad Robusta. En Venezuela, Arrieta (2004) obtuvo buen resultado en la captura de brocas logrando un promedio semanal de 124 adultos al utilizar extractos de cereza de café mezclado con aguardiente de Agave cocuy en proporción de 3:1. Aunque en Venezuela se ha venido trabajando con el uso de las trampas alcohólicas para el control de broca del café, aún no se ha determinado cual es el atrayente alcohólico más efectivo en la captura de adultos, la mejor altura de colocación de trampas, ni como 
fluctúa la población del insecto durante el período inter-cosecha.

A fin de evaluar la presencia de la broca en la comunidad del Choro, provincia Nor Yungas del Departamento de La Paz se propone trampas artesanales utilizando diferentes alcoholes como atrayentes alimenticios como una alternativa en el monitoreo y control de la broca del café y determinar la fluctuación poblacional de adultos de broca del café durante el período inter-cosecha

\section{Materiales y métodos}

Los ensayos se realizaron en la comunidad de Choro perteneciente al municipio de Coripata, segunda sección de la provincia Nor Yungas del departamento de La Paz, a una altitud comprendida entre 1060 a 4000 msnm y temperatura variable entre $11-26{ }^{\circ} \mathrm{C}$. (Gobierno Municipal de Coripata 2006).

Se diseñaron tres trampas caseras de botellas plásticas PET de 2 litros de capacidad, (fig. 1) y vasos plásticos descartables, i). Brocap basado en el diseño propuesto por la Fundación Salvadoreña para Investigaciones del Café del Salvador (PROCAFE de El Salvador, 2006) y por el Centro de Cooperación Internacional de Investigación Agronómico para el Desarrollo (CIRAD); ii). Trampas Caseras propuestas por (Fernández 2005) y iii). Trampas Yessica confeccionadas de acuerdo a la propuesta de la facultad de agronomía de la Universidad Central de Venezuela. (Fernández 2005).

Los atrayentes contenían 3 tipos de soluciones: i). 3:1 metanol-etanol. ii). 1:1 metanol-etanol. iii). 1:1:1 metanol-etanol-cereza fresca de café licuado, se distribuyeron en frascos de $50 \mathrm{ml}$ que tenían en la tapa un agujero de unos $3 \mathrm{~mm}$ de diámetro para facilitar la evaporación de la mezcla, En las trampas caseras se colgaron dentro de las botellas, a la altura de las ventanas que previamente se habían abierto para permitir la difusión de los mismos (Fig. 3). Las trampas Yessica los frascos se colgaron dentro de los vasos y suspendidos por una pita de cáñamo que estaba sujetado a la tapa de la trampa. Las Brocap los frascos fueron insertados en medio de las dos aletas y sujetados de la tapa por medio de una pita de cáñamo.

En el fondo de todas las trampas se depositó una solución jabonosa para romper la tensión superficial del agua e impedir el escape de los insectos capturados.

Se preparó 45 unidades experimentales (trampas), 4 de cada mezcla alcohólica, que hacen un total de 12 por tipo de trampa y 9 testigos que contenían agua, distribuidas al azar en 1,5 ha de terreno de café (Coffea arabica L.) var. Caturra Amarillo, a una altura de 1,20 m del cafeto, desde agosto a noviembre que es la etapa poscosecha.

Las trampas se revisaron el 08, 18, 28 de agosto, 07, 17, 27 de septiembre 07, 17, 27 de octubre y 06 de noviembre (2007), registrando el número de brocas (H. hampei) capturadas por trampa, se cambió el agua jabonosa y se aumentó la cantidad de los atrayentes a cada unidad experimental. La identificación de cada espécimen se la realizo utilizando un esteromicroscopio Olimpus, la cuantificación de brocas fue utilizando el indicador numero de especimenes capturados cada 10 días por unidad experimental (trampa).

Las capturas de broca por trampa en cada bloque y fecha de revisión se compararon estadísticamente a través de un análisis de varianza (ANVA).

Como resultado del análisis estadístico se obtuvo un coeficiente de variación muy alto (hasta $174,33 \%$ ), valor que no se ajusta al parámetro 
permitido (CV $=1 \%$ a $29 \%)$. Por esta razón se dio una alternativa de transformación de datos para que el coeficiente de variación del análisis estadístico esté dentro del rango aceptado. Para ello se realizó una transformación logarítmica de valores.

\section{Resultados}

Tabla 1 Captura por trampa Casera de adultos de broca del café (Hypothenemus hampei) con tres atrayentes (mezclas alcohólicas) durante 10 capturas consecutiva en la comunidad Choro municipio de Coripata segunda sección de la provincia Nor Yungas del Departamento de La Paz, Bolivia

\begin{tabular}{|c|c|c|c|c|c|c|c|c|c|c|c|c|}
\hline \multirow{2}{*}{$\begin{array}{l}\text { Captura } \\
\text { (fecha) }\end{array}$} & $\mathrm{Nr}$ & Min. & Máx. & Media \pm SD & $\mathrm{Nr}$ & Min. & Máx. & Media \pm SD & $\mathrm{Nr}$ & Min. & Máx. & Media \pm SD \\
\hline & \multicolumn{4}{|c|}{ Met - et 3:1 } & \multicolumn{4}{|c|}{ Met - et 1:1 } & \multicolumn{4}{|c|}{ Met - et - CFCL 1:1:1 } \\
\hline $08 / 08$ & 65 & 11 & 42 & $21.7 \pm 17.6$ & 96 & 5 & 35 & $24 \pm 13.2$ & 324 & 5 & 181 & $81.8 \pm 2.2$ \\
\hline $18 / 08$ & 175 & 12 & 114 & $43.8 \pm 47.3$ & 319 & 37 & 142 & $97.8 \pm 51.1$ & 404 & 15 & 317 & $100.3 \pm 145.6$ \\
\hline $28 / 08$ & 601 & 49 & 460 & $200.3 \pm 225.9$ & 412 & 52 & 190 & $103 \pm 60.2$ & 665 & 24 & 500 & $166.3 \pm 223.6$ \\
\hline 07/09 & 1502 & 217 & 465 & $375.5 \pm 113.6$ & 4225 & 278 & 2820 & 1056.3 & 1642 & 164 & 890 & $410.5 \pm 325.7$ \\
\hline $17 / 09$ & 7864 & 851 & 5356 & $1966 \pm 2265.7$ & 2910 & 362 & 1495 & $727.5 \pm 525.2$ & 3244 & 406 & 2030 & $811 \pm 823.4$ \\
\hline $27 / 09$ & 2714 & 156 & 1506 & $678.5 \pm 631.9$ & 1402 & 95 & 929 & $350.5 \pm 391.4$ & 1598 & 106 & 1012 & $399.5 \pm 413.2$ \\
\hline $07 / 10$ & 1420 & 156 & 580 & $355 \pm 188$ & 855 & 60 & 538 & $213.8 \pm 220.3$ & 680 & 60 & 350 & $170 \pm 128.3$ \\
\hline $17 / 10$ & 6251 & 450 & 2940 & $1562.8 \pm 1242.8$ & 1357 & 167 & 666 & $339.3 \pm 222.4$ & 1425 & 98 & 526 & $356.3 \pm 186.7$ \\
\hline $27 / 10$ & 8443 & 1072 & 3055 & $2110.8 \pm 1009.7$ & 1858 & 236 & 790 & $464.5 \pm 312.3$ & 2708 & 506 & 770 & $677 \pm 117$ \\
\hline $06 / 11$ & 5110 & 633 & 2211 & $1275 \pm 757.7$ & 1509 & 217 & 548 & $377.3 \pm 160$ & 1798 & 490 & 580 & $449.5 \pm 149.5$ \\
\hline
\end{tabular}

Met (metanol) et (etanol) CFCL (cereza fresca de café licuado). (3:1; 1:1) relación de las mezclas metanol etanol (1:1:1) mezcla Met-et-CFCL, El total de adultos ( $\mathrm{Nr}$ ) resulta de la suma de las brocas capturadas de 4 trampas desde el 08 de agosto al 06 de noviembre (2007). La Media de adultos de 4 trampas para cada tratamiento ( \pm desviación estándar) de broca capturada/trampa/10 días.

Nr. Numero de individuos. Min. (Mínimo de individuos), Max. (Máximo de individuos) SD Desviación estándar. 
Tabla 2 Captura por trampa Yessica de adultos de broca del café (Hypothenemus hampei) con tres atrayentes (mezclas alcohólicas) durante 10 capturas consecutiva en la comunidad Choro municipio de Coripata segunda sección de la provincia Nor Yungas del Departamento de La Paz, Bolivia

\begin{tabular}{|c|c|c|c|c|c|c|c|c|c|c|c|c|}
\hline \multirow{2}{*}{$\begin{array}{l}\text { Captura } \\
\text { (fecha) }\end{array}$} & $\mathrm{Nr}$ & Min. & Máx. & Media \pm SD & $\mathrm{Nr}$ & Min. & Máx. & Media \pm SD & $\mathrm{Nr}$ & Min. & Máx. & Media \pm SD \\
\hline & \multicolumn{4}{|c|}{ Met - et 3:1 } & \multicolumn{4}{|c|}{ Met - et 1:1 } & \multicolumn{4}{|c|}{ Met - et - CFCL 1:1:1 } \\
\hline 08/08 & 49 & 4 & 25 & $12.3 \pm 9.1$ & 35 & 6 & 12 & $8.8 \pm 2.8$ & 46 & 2 & 26 & $11.5 \pm 10.5$ \\
\hline $18 / 08$ & 251 & 16 & 111 & $62.8 \pm 40.9$ & 205 & 18 & 112 & $51.3 \pm 42.8$ & 121 & 19 & 43 & $30.3 \pm 10.2$ \\
\hline $28 / 08$ & 165 & 18 & 87 & $41.3 \pm 31$ & 299 & 28 & 179 & $74.8 \pm 71.2$ & 179 & 20 & 71 & $44.5 \pm 21.6$ \\
\hline 07/09 & 1089 & 23 & 598 & $272.3 \pm 248.7$ & 1769 & 207 & 855 & $442.3 \pm 295.8$ & 1049 & 150 & 486 & $262.3 \pm 180.7$ \\
\hline $17 / 09$ & 1919 & 45 & 1030 & $479.8 \pm 413.3$ & 1453 & 15 & 618 & $363.3 \pm 257.5$ & 1401 & 95 & 565 & $350.3 \pm 214.7$ \\
\hline $27 / 09$ & 561 & 86 & 268 & $140.3 \pm 85.8$ & 811 & 88 & 388 & $202.8 \pm 136$ & 360 & 50 & 148 & $90 \pm 44.8$ \\
\hline $07 / 10$ & 397 & 56 & 144 & $99.3 \pm 46.7$ & 438 & 66 & 200 & $109.5 \pm 61.2$ & 526 & 66 & 240 & $131.5 \pm 75.7$ \\
\hline $17 / 10$ & 1089 & 46 & 465 & $272.3 \pm 214.6$ & 917 & 148 & 309 & $229.3 \pm 76.5$ & 144 & 56 & 272 & $144 \pm 91.7$ \\
\hline $27 / 10$ & 1301 & 50 & 732 & $325.3 \pm 289.7$ & 1256 & 122 & 630 & $314 \pm 233.5$ & 1045 & 206 & 300 & $261.25 \pm 39.8$ \\
\hline $06 / 11$ & 670 & 30 & 314 & $167.5 \pm 116.5$ & 947 & 112 & 487 & $236.8 \pm 172.3$ & 503 & 105 & 152 & $125.8 \pm 22.2$ \\
\hline
\end{tabular}

Met (metanol) et (etanol) CFCL (cereza fresca de café licuado). (3:1; 1:1) relación de las mezclas metanol etanol (1:1:1) mezcla Met-et-CFCL. El total de adulto (Nr) resulta de la suma de las brocas capturadas de 4 trampas desde el 08 de agosto al 06 de noviembre (2007). La media de adultos de 4 trampas para cada tratamiento ( \pm desviación estándar) de broca capturada/trampa/10 días.

Nr. Numero de individuos. Min. (Mínimo de individuos), Max. (Máximo de individuos) SD Desviación estándar.

Tabla 3 Captura por trampa Brocap de adultos de broca del café (Hypothenemus hampei) con tres atrayentes (mezclas alcohólicas) durante 10 capturas consecutivas en la comunidad Choro municipio de Coripata segunda sección de la provincia Nor Yungas del Departamento de La Paz, Bolivia

\begin{tabular}{|c|c|c|c|c|c|c|c|c|c|c|c|c|}
\hline \multirow{2}{*}{$\begin{array}{l}\text { Captura } \\
\text { (fecha) }\end{array}$} & $\mathrm{Nr}$ & Min. & Máx. & Media \pm SD & $\mathrm{Nr}$ & Min. & Máx. & Media \pm SD & $\mathrm{Nr}$ & Min. & Máx. & Media \pm SD \\
\hline & \multicolumn{4}{|c|}{ Met - et 3:1 } & \multicolumn{4}{|c|}{ Met - et 1:1 } & \multicolumn{4}{|c|}{ Met - et - CFCL 1:1:1 } \\
\hline 08/08 & 29 & 4 & 10 & $7.3 \pm 3.2$ & 13 & 0 & 8 & $3.3 \pm 3.4$ & 102 & 20 & 33 & $25.5 \pm 5.4$ \\
\hline $18 / 08$ & 219 & 10 & 95 & $54.8 \pm 42.6$ & 53 & 5 & 21 & $13.3 \pm 6.7$ & 284 & 29 & 105 & $71 \pm 36.6$ \\
\hline $28 / 08$ & 128 & 12 & 46 & $32 \pm 15.8$ & 129 & 0 & 123 & $32.3 \pm 60.6$ & 341 & 6 & 147 & $85.3 \pm 58.5$ \\
\hline 07/09 & 860 & 63 & 552 & $215 \pm 228.3$ & 2590 & 81 & 2134 & $647.5 \pm 992.5$ & 5986 & 93 & 5220 & $1496.5 \pm 2485.5$ \\
\hline $17 / 09$ & 1103 & 141 & 400 & $275.8 \pm 119.2$ & 1515 & 95 & 1007 & $378.8 \pm 422.3$ & 2365 & 150 & 1090 & $591.3 \pm 404.8$ \\
\hline $27 / 09$ & 868 & 80 & 530 & $217 \pm 217.8$ & 916 & 25 & 776 & $229 \pm 365.5$ & 637 & 66 & 426 & $159.3 \pm 178$ \\
\hline $07 / 10$ & 498 & 45 & 228 & $124.5 \pm 77.3$ & 327 & 35 & 150 & $81.8 \pm 51.5$ & 467 & 58 & 279 & $116.8 \pm 108.3$ \\
\hline $17 / 10$ & 801 & 46 & 422 & $200.3 \pm 161.2$ & 436 & 32 & 212 & $109 \pm 87$ & 606 & 58 & 232 & $151.5 \pm 71.6$ \\
\hline $27 / 10$ & 1165 & 262 & 345 & $291.3 \pm 36.7$ & 617 & 45 & 246 & $154.3 \pm 83.8$ & 1180 & 245 & 369 & $295 \pm 52.5$ \\
\hline $06 / 11$ & 685 & 85 & 292 & $171.3 \pm 90.2$ & 540 & 65 & 250 & $135 \pm 81.9$ & 701 & 56 & 262 & $176.3 \pm 89.5$ \\
\hline
\end{tabular}

Met (metanol) et (etanol) CFCL (cereza fresca de café licuado). (3:1; 1:1) relación de las mezclas metanol etanol (1:1:1) mezcla Met-et-CFCL. El total de adulto (Nr) resulta de la suma de las brocas capturadas de 4 trampas desde el 08 de agosto al 06 de noviembre (2007). La Media de adultos de 4 trampas para cada tratamiento ( \pm desviación estándar) de broca capturada/trampa/10 días.

Nr. Numero de individuos. Min. (Mínimo de individuos), Max. (Máximo de individuos) SD Desviación estándar. 
Tabla 4 Captura por trampa Casera, Yessica, Brocap de adultos de broca del café (Hypothenemus hampei) con tres atrayentes (mezclas alcohólicas) durante 10 capturas consecutivas en la comunidad Choro municipio de Coripata segunda sección de la provincia Nor Yungas del Departamento de La Paz, Bolivia

\begin{tabular}{|c|c|c|c|c|c|c|c|c|c|c|c|c|}
\hline Trampa & $\mathrm{Nr}$ & $\begin{array}{c}\text { Mi } \\
\text { n. }\end{array}$ & Máx. & Media \pm SD & $\mathrm{Nr}$ & Min. & Máx. & Media \pm SD & $\mathrm{Nr}$ & Min. & Máx. & Media \pm SD \\
\hline (Unidad) & \multicolumn{4}{|c|}{ Met - et 3:1 } & \multicolumn{4}{|c|}{ Met - et 1:1 } & \multicolumn{4}{|c|}{ Met - et - CFCL 1:1:1 } \\
\hline Casera & 34145 & 11 & 1072 & $3414.5 \pm 3227.7$ & 14943 & 96 & 4225 & $1494.3 \pm 1270.9$ & 14488 & 324 & 3244 & $1448.8 \pm 974.8$ \\
\hline Jessica & 7491 & 49 & 1919 & $749.1 \pm 591.5$ & 8130 & 36 & 1769 & $813 \pm 570.4$ & 5310 & 46 & 1401 & $531 \pm 476.8$ \\
\hline Brocap & 6356 & 29 & 1165 & $617.6 \pm 401.5$ & 7136 & 13 & 2590 & $713.6 \pm 789.5$ & 12669 & 102 & 5986 & $1266.9 \pm 1778.2$ \\
\hline Testigo & 73 & 0 & 23 & $2.4 \pm 4.2$ & 79 & 0 & 20 & $2.6 \pm 4.1$ & 63 & 0 & 20 & $21.1 \pm 4.1$ \\
\hline
\end{tabular}

Met (metanol) et (etanol) CFCL (cereza fresca de café licuado). (3:1; 1:1) relación de las mezclas metanol etanol (1:1:1) mezcla Met-et-CFCL, testigo (agua) El total de adulto (Nr) resulta de la suma de las brocas capturadas de 12 trampas y 9 trampas del testigo desde el 08 de agosto al 06 de noviembre (2007). La Media de adultos de 12 trampas para cada tratamiento ( \pm desviación estándar) de broca capturada/trampa/10 días.

Nr. Numero de individuos. Min. (Mínimo de individuos), Max. (Máximo de individuos) SD Desviación estándar.

Tabla 5 Resultado de análisis de varianza de trampas (Unidades) Casera, Yessica, Brocap en la captura de brocas adultas broca del café (Hypothenemus hampei) en las diez evaluaciones consecutivas (cada diez dias) en la comunidad Choro municipio de Coripata segunda sección de la provincia Nor Yungas del Departamento de La Paz, Bolivia

\begin{tabular}{lcrrrr}
\hline \multicolumn{1}{c}{ Fuente de variación } & Grados de libertad & Suma de cuadrados & Cuadrado medio & F calculada & Probabilidad \\
\hline Trampa & 2 & 45.0826 & 22.5413 & $45.08^{* *}$ & $<0.0001$ \\
Atrayente & 3 & 453.9888 & 151.3296 & $302.61^{* *}$ & $<0.0001$ \\
Trampa*atrayente & 6 & 16.7177 & 2.7863 & $5.57^{* *}$ & $<0.0001$ \\
Fecha & 9 & 215.7545 & 23.9727 & $47.94^{* *}$ & $<0.0001$ \\
Trampa*fecha & 18 & 7.2688 & 0.4038 & 0.81 NSE & 0.6915 \\
Atrayente*fecha & 27 & 63.6 & 2.3556 & $4.71^{* *}$ & $<0.0001$ \\
Trampa*atrayente*fecha & 54 & 15.8729 & 0.2939 & 0.59 NSE & 0.9905 \\
Error & 330 & 165.0249 & 0.5001 & & \\
Total & 449 & 1019.488 & & & \\
\hline
\end{tabular}

Coeficiente de variabilidad $15.77 \%$.** Representa una diferencia estadística. NSE No representa diferencia estadística. 


\section{Discusión}

En los países productores de café la broca del café Hypothenemus hampei (Ferrari): (Coleoptera: Curculionidae: Scolytinae), es considerado el principal insecto plaga, en los países del África ecuatorial (López-Vaamonte et al 1997, Damon 2000). Insectos hembras que perforan el fruto ocasionado daños como la caída de fruto y perdida de peso del grano de café. (Wegbe et al 2003)

En la comunidad del Choro, municipio de Coripata, al ser una zona que practica la cafecultura, no se han realizado trabajos que aborden este tipo de temáticas debido a los costos que involucra de ahí que constituye una problemática socio-económica de repunte.

Los resultados obtenidos en las 10 capturas de agosto a noviembre (2007) utilizando tres tipos de trampas (unidades) construidas artesanalmente de botellas de bebidas gaseosas y vasos descartables de plástico, se observa una fluctuación poblacional de que, la media ( \pm desviación estándar) de broca capturadas fue menor (Casera 21,7 $\pm 17,6 ; 24 \pm 13,2$; 81,8 \pm 2 ) con mezclas de M:E, 3:1; M:E; 1:1; M:E:CFCL; 1:1:1) (tabla 1) y conforme paso el tiempo éste siguió una tendencia a incrementarse de tal manera que las capturas máximas fueron en fechas intermedias de la mezcla (3.1) $(1966 \pm 2265,7 ; \quad 1562,8 \pm 1242,8 ; \quad 2110,8 \pm 1009,7)$, situación similar que se observa en las mezclas $(1: 1 ; 1: 1: 1)$ (tabla 1$)$ lo que quiere decir que hay factores ambientales, color de la trampa, altura $\mathrm{u}$ otros, que esté trabajo no abordo y han sido determinantes.
Las trampas Yessica, las capturas de inicio, media ( \pm error estándar) $(12,3 \pm 9,1 ; 8,8 \pm 2,8 ; 11,5 \pm 10,5)$ con mezcla $3: 1 ; 1: 1 ; 1: 1: 1$ (tabla 2 ) se observa, una tendencia a ser homogénea en las capturas periódicas que se realizó, posiblemente la forma de la trampa, su altura han sido condicionantes llegando a $\quad(167,5 \pm 116,5 ; \quad 236,8 \pm 172,3$; $125,8 \pm 22,2)$, observándose en el tiempo de capturas dos picos altos de capturas de brocas, $(07 / 09 ; 17 / 09)$ para la mezcla $(3: 1)$ y $(27 / 10)$ para las mezclas $(1: 1,1: 1: 1)$, observándose el resto de los tiempos de muestreo una tendencia a la homogeneidad. (tabla 2).

Las trampas (Brocap 7,3 $\pm 3,2 ; 3,3 \pm 3,4 ; 25,5 \pm 5,4$ ) con mezclas $3: 1 ; 1: 1 ; 1: 1: 1$ ), siendo la trampa que menos captura de brocas en su inicio logra, en comparación con las trampas Casera y Yessica, posiblemente la disposición de la trampa o color fue el factor que determino estos resultados, llegándose a la fecha ultima de capturas con valores de $(171,3 \pm 90,2 ; 135 \pm 81,9 ; 176,3 \pm 89,5$ para las tres mezclas de atrayentes utilizadas) (tabla 3), y si observamos alo largo del tiempo de monitoreo no se observan capturas máximas que superen a las trampas Caseras.

Cuando comparamos las tres tipos de trampas con los atrayentes utilizadas en este trabajo, se observa que la trampa Casera, con la mezcla (3:1) resulta ser la alternativa para el monitoreo de la broca del cafeto, en relación a la Yessica y Brocap, e inclusive el testigo que contenía agua como solución atrayente (tabla 4), habiéndose logrado 
una media total ( \pm desviación estándar) $(3414,5 \pm 3227,7$ con la mezcla $3: 1)$ siendo cerca de cinco veces mayor su probabilidad de captura en relación a la Yessica y Brocap, inclusive con las mezclas atrayentes $(1: 1 ; 1: 1: 1)$ la trampa Casera supera a esta dos. Nuestros datos son diferentes a (Fernández \& Cordero 2005) quien evaluó durante diez semanas en Venezuela, cuyas condiciones ambientales y altitud son diferentes, y el tiempo de evaluación de los meses de febrero a mayo, además de aplicar otros indicadores en sus evaluaciones como ser el tiempo de captura, que lo realizó semanalmente. En tanto (Barrera et al 2008) evaluó de febrero a marzo, utilizando otros tipos de trampas (ETOTRAP y ECOIAPAR), donde determino la población fluctuante de broca diferente a nuestros resultados, pero este autor practico métodos de captura diferente al nuestro.

La discordancia de nuestros resultados con estos autores se debe a que la época muestreo no es igual, puede que los factores ambientales estén influyendo en al biología de la broca del cafeto, dado que no hay trabajos en el área, grado de infestación en las diferentes épocas del años, si a esto se suma el uso de controladores biológicos, la aplicación de plaguicidas, entre otros, además las trampas de color no siempre dieron resultados satisfactorios (Barrera et al 2006).

García et al (2004) comparó las trampas (Brocap y Fiesta) cuyos resultados mostraron que en cuatro meses y 16 capturas semanales de mayo a agosto y 16 trampas por modelo, la trampa Brocap capturó 679107 brocas (2653 brocas/trampa/semana), 2.44 y 3.17 veces más que ECOIAPAR y Fiesta, respectivamente. La "saturación" de las trampas artesanales con brocas fue la causa de la diferencia, nuestros datos no se aproximan a los de este autor, por los tiempos y las capturas que han sido minuciosas, en tanto nuestras capturas fuerón cada diez, dias, pero concordamos con estos investigadores, pues la saturación de brocas pudo haberse dado, de ahí que el receptáculo de brocas tendríamos que considerar para futuros trabajos, en tanto las trampas Brocap, quizá el cambiar de color sea una de las primeras medidas que tendríamos que revisar en las próximas experiencias.

Todos estos aspectos nos tienen que llevar a la reflexión, que la broca del cafeto es un problema de orden socio-económico, se requiere plantear alternativas de control de esta plaga, de ahí que este trabajo requiere un seguimiento a lo largo de la época de producción, ínter-cosecha, etc, a fin de evaluar si esta alternativa permite desarrollar mejoras en la producción del café.

Si bien es necesario completar estudios acerca de los resultados obtenidos acerca de las trampas diseñadas para la captura de la broca del cafeto en el Choro municipio de Coripata provincia Nor Yungas del departamento de La Paz, Bolivia, los resultados obtenidos en este trabajo indicarían que los adultos infestan los cultivos de café atraídos por los olores del alcohol y las trampas casera serian una alternativa, debido a que los aspectos evaluados en relación a la trampa y el atrayente de 
estas especie indican ser un potencial de plaga en los cultivos de café.

\section{Conflictos de interés}

Esta investigación recibió financiamiento parcial de USAID/Bolivia, el Instituto de Investigaciones y no presenta conflictos de interés.

\section{Agradecimientos}

Los autores agradecen a los productores cafetaleros de la Comunidad del Choro, al personal del Laboratorio de Entomología de la Carrera de Agronómica. A la Unidad Académica Campesina Carmen Pampa por el financiamiento de la investigación. Al Instituto de Investigaciones del la Unidad Académica Campesina Carmen Pampa.

\section{Literatura citada}

Aristizábal LF, Salazar H, Mejía CG. Cambios en la adopción de los componentes de manejo integrado de la broca del café, Hypothenemus hampei (Coleoptera: Scolytidae) a través de metodologías participativas. Revista Colombiana de Entomología. 2002; 28:153160.

Arrieta A. Evaluación de trampas y atrayentes para la captura de la broca del café Hypothenemus hampei (Ferrari), 1867 (Coleoptera: Scolytidae) en la localidad de El Jabón, municipio Torres, Estado Lara. [Tesis de Licenciatura]. Barquisimeto, Venezuela:
Universidad Centroccidental Lisandro Alvarado; 2004.

Barrera JF, Herrera J, Chiu M, Gómez J, Valle J. The one window trap (ECOIPAR) captures more coffee berry borer Hypothenemus hampei than the three windows trap (ETOTRAP) Entomología Mexicana. 2008; 7: 619-624.

Barrera JF, Herrera J, Villacorta A, Garcia H, Cruz L. Methanol-ethanol traps for detection, monitoring and control of the coffee berry borer Hypothenemus hampei. Simposio sobre Trampas y atrayentes en detección, monitoreo y control de plagas de importancia económica. JF Barrera \& Monoya P. (eds.). Sociedad Mexicana de Entomología y El Colegio de la Frontera Sur Manzanillo, Colima, México, 7183. ISBN 970-9712-28-4; 2006.

Barrera JF, Herrera J, Villacorta A, Garcia H, Cruz L. Trampas de metanol-etanol para detección, monitoreo y control de la broca del café Hypothenemus hampei. Simposio Trampas $\mathrm{y}$ atrayentes en detección, monitoreo y control de plagas de importancia económica. El colegio de la Frontera Sur (ECOSUR) Tapachula, Chiapas, México; 2006. [Consultado: 12 ene. 2008] Disponible en: http://www2.tapecosur.edu.mx/mip/pdf/Simposio_Trampas 20 06.pdf.

Belpaire C, Chorquina V. Experiencias de Control Biológico de Plagas Agrícolas, vol. I. La Paz, Bolivia; Editorial Instituto de Ecología; 1998. 
Chuquimia G. Manual para la Producción Ecológica de Café, FECAFEB Editorial Hepta Producciones. La Paz, Bolivia; 1996.

Cuba N. Manual para el cultivo de café en los Yungas. Carmen Pampa-La Paz, Bolivia: Universidad católica Boliviana, Unidad Académica Campesina Carme Pampa, 2003: p. 7.

Damon A. A review of the biology and control of the berry borer, Hypothenemus hampei (Coleoptera: Scolytidae). Bull Entomol Res. 2000; 90: 453- 465.

Fernández S, Cordero J. Evaluación de atrayentes alcohólicos en trampas artesanales para el monitoreo y control de la broca del café, Hypothenemus hampei (Ferrari). Revista Digital del Instituto Nacional de Investigaciones Agrícolas (INIA). Bioagro. 2005; 17(3), 143-148.

Fernández S. Trampas artesanales con alcoholes: una estrategia fácil de utilizar para el control de broca del café. Revista Digital del Centro Nacional de Investigaciones Agropecuarias de Venezuela [CENIAP] 2006; (8). Maracay, Aragua-Venezuela. [Consultados 17 nov. 2006] Disponible en: $\mathrm{http} /$ www.ceniap.gov.ve/ceniaphoy/articulos/n 8/arti/fernandez_s/fernandez_s.htm.

Fundación Salvadoreña para la Investigación del Café. Manejo integrado de la broca del café. (IICA/PROMECAFE). El Salvador: PROCAFE; 2006. [Consultado 28 ene 2008] Disponible en: http://www.iica.org.gt/promecafe/boletines/mib /boletin2_mib.pdf.

García-Verdugo H, Barrera JF, Pinson E, Valle FJ, Herrera J. Comparación de tres tipos de trampas para la captura de la broca del café. En: Resúmenes del I Congreso Internacional sobre Desarrollo de Zonas Cafetaleras. 2004 oct. 6-8; Tapachula, Chiapas, México; 2004; p. 45.

Gobierno Municipal de Coripata. Plan de Desarrollo Municipal (PDM). 2006-2010. Segunda Sección de la Provincia Nor Yungas. La Paz, Bolivia: Servicios, Proyectos e inversiones COSEPI; 2006.

López-Vaamonde C, Baker PS, Cock M, OrozcoHoyos J. Informe sobre Phymastichus coffea (Hymenoptera: Eulophidae, Tetrastichinae), un agente de control biológico contra Hypothenemus hampei (Coleoptera: Scolytidae) en Colombia. Commonwealth Agricultural Bureau. London, UK; 1997.

Minitab. User's guide: statistical software. State College, PA. Minitab Inc. 2007. Version 15.

Ramírez GY, Mora M. Boletín informativo: la broca del fruto del café nos amenaza. San José, Costa Rica: [ICAFE] Instituto del café de Costa Rica, 2001; p. 70.

Velasco HB, Ruiz B, Díaz S, Llavén JM, Velásquez AF. Respuesta de la broca del fruto Hypothenemus hampei Ferr. a extractos de cerezas de café utilizados como atrayentes en Tepatlaxco, Veracruz, México: Universidad de Chapingo; 1997. [Consultado 19 ago. 2005] 
Disponible en http://www.

ecologia.edu.mx/sigolfo/p.htm.

Wegbe K, Cilas C, Decazy B, Alauzet C, Dufour

B. Estimation of production losses caused by the coffee berry borer (Coleoptera: Scolytidae) and calculation of an economic damage threshold in Togolese coffee plots. J. Econom. Entomol. 2003; 96: 1473-1478. 